\title{
DE LA SOCIÉTÉ DU RISQUE AUX CONDUITES À RISQUE
}

Jean-Martin RABOT *

\section{Asepsie sociale, risques et conduites à risque}

Face à des sociétés soumises à des processus de changement rapides et constants, Face à des sociétés soumises à des pherché à leur adosser un qualificatif, ne seraitles sociologues ont depuis toujours cherché à leur adosser un qualificatif, ne seraitce que pour leur conférer un semblant de stabilité, dans un monde qui se veut incertain et qui se vit dans l'incertitude. Ce qui est nommé est en effet d'emblée plus compréhensible et plus maîtrisable, même lorsque les dénominations traduisent la labilité, l'inconstance et le mouvement. Aussi les a-t-on désignées comme étant labilité, l'inconstance et le mouvement. Aussi jours, en raison de la part grandisindustrielles, conflictuelles, liquides, etc. De nos jours, en raison de la part grandissante qu'y prennent les accidents en tout genre, les sociétés sont perçues comme étant à risque. Des fléaux en tout genre menacent la vie de l'individu et de l'espece des catastrophes environnementales, en raison d'une mauvaise gestion des ressources, reflets de pratiques économiques et politiques peu scrupuleuses, mais auss des désastres qui touchent à une gamme toujours plus vaste de phénomènes, allant des accidents domestiques aux dérives du terrorisme.

Une double constatation guide la présente réflexion : premièrement, à mesure Ue les sociétés sont mieux gérées, qu'elles sont bureaucratisées (Max Weber, Bruno que directoriales (James Burnham), appareillées (Jacques Beau-

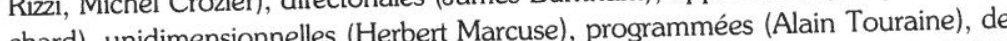
chard), unidimensionnelles (Hichel Mafsurveillance ou de contrôle (Michel Foucault), aseptisées et hygenisées (Mich socíté fesoli), le risque a tendance à y proliférer; deuxiemement, à mesure que les sociétes contrôlent le risque, d'une part au moyen de la prévision, c'est-à-dire de la généralicontrobilistes et de statistiques publiques, dont le Le Petit

* Université du Minho, Braga, Portugal. 

Robert nous dit qu'il s'agit de l'« étude méthodique des faits sociaux, par des procédés numériques (classements, dénombrements, inventaires chiffrés, recense ments), destinée à renseigner et aider les gouvernements ", d'autre part au moyen de la prévention avec le développement de mesures prophylactiques et répresside la prévention avec le systèmes de protection efficaces, nous assistons à une ves, et enfin au món délibérément recrudescence des conduites à risque, c' est-à-dire de com
et ostentatoirement dangereux pour soi et pour autrui.

Nous serons ainsi amenés à comprendre la signification sociologique des conNous serons ainsi am les sociétés postmodernes, en particulier dan duites à risque qui se multiplient dans les sociétés postmodernes, en particulier dans les milieux juvéniles. Ces conduites ne se rapportent nullement à la faillite de sociétés incapables de promouvoir des systèmes de santé et d'éducation efficaces ou encore de produire un modèle familial apte à inculquer et à transmettre des valeurs encore de produire un modéle tamil conduites représentent précisément autant de respectueuses de la vie. De telles conduites représentent précisérés, qui érigent la " folies sociétales » saines dans des sociétés hautement rationalisees, qui érigent la prévision, la prévention et la protection en règle et l'hygiénisme en mode de vie. Les conduites à risque témoignent ainsi de l'ambiance quotidienne qui prévaut dans la conduites a risque témoignent ainsi daisir prend le pas sur le principe de réalité. Elles postmodernité, où le principe de plaisir prend le pas sur lécessité qu'a l'individu de sont le signe de l'irréductibilité de l'être-ensemble, de la nécessité qu'a l'individu de sortir de lui pour s'abandonner au destin et s'ouvrir aux autres. Elles nous confrontent à la sensation de l'irrémédiable et de l'absolu, posent certes «l'hypothèse non négligeable de mourir " 1 , mais nous exposent aussi à une expérience de la radicalité négligeable de mourir " ', mais nous exponer plus de solidité aux fondements de qui nous montre "que le seul moyen de donner plus de solidié aux fondements de la société [est] de donner un rôle au côté obscur qui sous-tend toutes les choses humaines, à l'excès d'énergie que tout organisme possède face à l'exigence de survie pure et simple»2

\section{Les conduites à risque comme antidote au processus de sécurisation et d'assurantialisation de la vie}

Nont les adversités de la vie Nombreuses comme les étoiles, les dieux et les dén. qui nous menacent tout au long de notre existence. Paradoxalement, celles-ci résultent le plus souvent des avancées scientifiques et techniques qui se devaient pourtant d'arracher l'homme aux malheurs de l'aléa et de le mener à l'empyree du bonheur, dans une perspective où « tous les ordres de progrès sont supposés liés, du matériel au spirituel, du cognitif au moral »3.

À cet égard, il suffit de prendre pour exemples Tchernobyl, la maladie du sida ou celle de Kreutzfeld-Jacob qui rappellent sans cesse à l'homme sa condition de ou celle de Kreutrelde inévitable des accidents de la nature et de la civilisation,

D. Le Breton, La peau et la trace. Sur les blessures de soi, Paris, Métailié, 2003, p. 10 2. G. da Empoli, La peste et l'orgie, Paris, Grasset, 2006, p. 18.

3. P.-A. Taguieff, Du progrès. Biographie d'une utopie moderne, Paris, E.J.L, 2001, p. 67.

Sociétés $n^{\circ} 114-2011 / 4$ ceux que Pareto appelait les vertueux ont mis sur pied des campagnes de préven tion, des politiques sociales plus égalitaires, des programmes éducatifs mieux adapés aux coutumes du temps et destinées aux populations les plus vulnérabes, indigents, comme l'on disait autrefois. Ainsi, nous serions en mesure de généraliser des modèles culturels guidés par les critères exclusifs de la raison et de la tempérance. Il s'agirait d'user de tout sans abuser de rien. Une attention particulière est donnée à l'éducation sexuelle, aux pratiques alimentaires saines, à la promotion de la santé, à la lutte contre les discriminations sociales, au combat contre l'ignorance et l'obscurantisme. On pourrait même se demander si les dictatures d'antan qui s'exerçaient au nom du pouvoir divin ou de celui de la souveraineté des peuples ne s'exercent pas de nos jours au nom de la salubrité publique et du respect de l'intégrité physique et psychologique de l'individu.

En tout état de cause, les campagnes de prévention, comme la campagne d'information lancée en France en 1999 par la mission interministérielle contre les drogues et la toxicomanie, intitulée "Savoir plus pour risquer moins", ont montré leurs limites, comme nous le rappelle Peretti-Watel : « de nombreuses expériences antérieures soulignent que la diffusion du savoir ne modifie pas forcément les conduites dites "à risque" " 4. "L'expérience humaine générale », selon l'heureuse formule de Weber, apporte un cinglant déni aux espérances fondées sur la croyance en un progrès continu conduisant à la domination de la nature et à l'équarrissage de l'humain. Les risques associés aux prises de risque délibérées sont là pour nous apprendre que ce que Kierkegaard appelait le "saut de la foi ", Pascal " le pari de l'existence de Dieu ", Foucault la " bio-politique ", Luhmann la " confiance ", Giddens la "sécurité ontologique ", Beck "lápartition rantialisation " ne sont que des panacées qui n'ont pas de réelle emprise sur la vie concrète des hommes. Ces derniers aiment à se frotter à l'inconnu, à l'incalculable,
à l'imprévisible, à l'insurmontable.

Une analyse perspicace de la réalité nous montre que les programmes de prévision et de prévention des risques, pour légitimes qu'ils soient d'un point de vue moral, du point de vue d'une raison bourgeoise qui fait de la " conservation de soi » et de l'intégration dans le tout social "par la prise de conscience des individus de leur intérêt » 5 une finalité ultime, relèvent de l'incantation. Il serait plus approprié de comprendre que les conduites à risque font écho au processus d'" effacement de l'expérience du moi ", corrélatif des progrès médicaux dans le domaine de la "prolongation artificielle de la vie " 6 dans le domaine également du traitement des maladies, et en particulier de l'utilisation généralisée des soins palliatifs qui aliènent la " conscience de l'existence de notre corporéité " ?. À ce propos, nous pouvons

4. P. Peretti-Watel, La société du risque, Paris, La Découverte \& Syros, 2001, p. 84. 5. M. Horkheimer, Eclipse de la raison, suivi de Raison et conservation de soi, Paris,
Payot, 1974, p. 203. Payot, 1974, p. 203

6. H.-G. Gadamer, Philosophie de la santé, Paris, Grasset \& Fasquelle et Mollat, 1998

7. Ibid., p. 84 
dire que les élucubrations faites à la veille des fêtes de Pâques de 2008 par l'archevêque de Pampelune, Fernando Sebastian Aguilar, ne semblent nullement ridicules, lorsque celui-ci affirma que la mort de Jésus sur la croix "a été une mort digne ", même si l'on n'a pas eu recours aux " soins palliatifs ".

Sociologiquement parlant, il est intéressant de noter que la prise irréfléchie de risques sur le plan personnel n'a pas diminué avec l'augmentation des tragédies sociales, à l'instar des guerres, des épidémies, des accidents nucléaires. C'est même le contraire qui se vérifie. Cela est dû en partie au processus de " sécurisation » de l'existence par des systèmes de protection qui ont étendu leurs tentacules à l'ensemble de la société. Plus rien n'échappe au domaine des assurances qui ont banalisé la notion de risque, comme l'a montré magistralement François Ewald dans son étude sur la sociogenèse de l'État providence : le travail, en première instance, mais aussi la vieillesse, la pauvreté, le handicap, le sport, l'entrepreneuriat et la vie elle-même. Il n'est jusqu'à la vie domestique qui n'ait pas été contaminée. Les accidents domestiques se sont métamorphosés en risques domestiques. Aux États-Unis, les plaintes contre les fabricants de cigarettes ou les marques de réfrigérants sont légions. Ces faits sont représentatifs du sens de l'évolution de l'État providence, avec la prolifération de systèmes de sécurité, dans lesquels le risque professionnel, tributaire de la loi civile, est transféré vers le risque social, corrélati du droit constitutionnel. Comme le souligne Ewald, le risque " passait d'un axe délictuel à un axe résolument contractuel. Mieux, il entraînait avec lui le passage de la problématique du délit à une problématique du contrat. La reconnaissance du "risque social" institue la rationalité du risque et de l'assurance au niveau d'une norme fondamentale, comme une source propre de juridiction $" 8$. En d'autres termes, avec la généralisation de la notion de risque, nous assistons à une sorte d'accaparement du social par les assurances : « le rapport social devait désormais épouser la forme de l'assurance " 9 . Et pire encore, avec l'expansion croissante de politiques de prévention de tous les risques, notre société relève de plus en plus d'un système totalitaire : "L'assurance devient obligatoire: on assure les individus, mais cela implique que l'on s'assure d'eux " 10 . Nous pouvons déduire de ces réflexions que les peurs ancestrales et ataviques ont été sublimées et que la violence qui était naturelle, socialement reconnue et acceptée, fait maintenant l'objet d'une dénégation et d'un refoulement.

Les temps changent, mais les problèmes demeurent intacts. De ce point de vue, les sociétés actuelles s'engouffrent dans les mêmes impasses que celles du Moyen Âge ou du début de la Renaissance : il s'agit de dénier le mal par des processus de substitution. Jean Delumeau a fait une description minutieuse de ces processus de conjuration des peurs qui hantaient nos aïeuls. La peur des guerres, des famines et des pestes était l'œuvre de l'Antéchrist qu'il s'agissait de démasquer coûte que coûte. De nos jours, nous continuons à nous voiler la face en refoulant la question de l'existence du mal. Non plus au moyen de "l'intrusion massive de

8. F. Ewald, L'État providence, Paris, Grasset, 1994, p. 333.

9. Ibid., p. 342. a théologie dans la vie quotidienne de la civilisation occidentale "11, mais par l'intromission de la science et de la technique dans nos vies. Le rationalisme a pris le relais de la théologie chrétienne dans ce processus de voilement et d'aliénation de l'expérience, comme l'a montré Weber. Il nous a conduit à croire " qu'à chaque instant nous pourrions, pourvu seulement que nous le voulions, nous prouver qu'il n'existe en principe aucune puissance mystérieuse et imprévisible qui interfère dans le cours de la vie ; bref que nous pouvons maîtriser toute chose par la prévision » 12 Aujourd'hui, grâce à des systèmes de prévision, de prévention et de protection efficaces, grâce aussi à une idéologie de la " sécurisation " et de l'" assurantialisation " de la vie, nous pensons pouvoir conjurer ces maux que sont les maladies, la criminalité, la violence, les carnages, la mort. Ces systèmes et cette idéologie ne protègent toutefois pas l'individu contre les " bas-fonds de la psyché "13, contre le désir insatiable qu'a l'homme de se confronter à l'expérience. En bref, les conduites à risque nous amènent à penser la vie, non pas comme un simple "devenir marchandise ", mais « comme existence, comme destin " 14.

On comprendra donc que les valeurs que suivent les individus sont rarement l'objet d'un consensus. La vérité est que les hommes peuvent préférer une existence moins longue, mais plus intensément vécue. La devise : "vivre vite, mourir jeune et faire un beau cadavre » semble être un exemple paradigmatique de la philosophie de vie de la jeunesse issue de la génération de James Dean. Existentiellement parlant, le dressage des êtres humains est tout à fait impossible, car il butte contre l'infinie variété des sensibilités humaines. L'homme est fait de bois noueux, comme l'a bien vu Kant. Nous pourrions même dire qu'il a raison de préférer le bon sens à l'imposition normative de la normalité par une raison impérieuse qui se veut universelle. Le bon sens pour lequel prime l'idée que l'incertitude est la "rançon de la liberté ", selon le mot du philosophe Élie Halévy 15. Le bon sens qui pour l'artiste consiste à représenter de manière récurrente des têtes de mort, des os, des squelettes pour se faire à l'idée de la finitude. La ruse de la vie ne consistet-elle pas à tourner la mort en dérision, à résister aux ravages que le temps exerce irrémédiablement sur nous? À profiter, même dangereusement et violemment, de chaque instant qui passe ? En défiant la mort, en l'intégrant à la vie de tous les jours, par le truchement d'actes que l'individu assume à ses risques et périls. Au moyen d'excès en tout genre, aussi inutiles qu'absurdes, au moyen de violences perpétrées contre les autres et exercées sur soi. La postmodernité se caractérise bien par la soumission de l'existence au sentiment tragique de la vie. Ce qui est en jeu dans ce défi métaphysique est précisément ce qui se joue dans l'érotisme: "l'approbation de la vie jusque dans la mort » 16 .

11. J. Delumeau, La peur en Occident (XIVe-XVIIle siècles), Paris, Hachette/Pluriel, 1985, 11. 40

12. M. Weber, Le savant et le politique, Paris, Union Générale d'Éditions, 1974, p. 70

12. M. Weber, Le savant et le politique, Paris, Union Générale de 155

14. M. Maffesoli, 152.

15. Cité par J. Ortega y Gasset, La révolte des masses, Paris, Gallimard, 1967, p. 175

16. G. Bataille, L'érotisme, Paris, Les Éditions de Minuit, 1985, p. 17. 


\section{Les conduites à risque comme facteur de socialité}

Ce que Norbert Elias appelait le processus de civilisation a notablement contribué à la domestication des hommes. La vie pulsionnelle a été réduite à sa portion congrue au profit d'un raffinement des mœurs qui est à la source de la rationalisation des actions. Les comportements déviants et la folie furent occultés, bannis et rejetés dans la sphère de l'irrationnel. On les accusa de relever de l'aliénation, en tant que carence de conscience, de liberté et de responsabilité individuelle. Or personne n'a mieux compris que Foucault que la folie interpelle l'homme, qu'elle maintient avec lui un rapport de réciprocité, qu'elle l'oblige à se confronter à sa vérité la plus essentielle comme à sa vérité finale: "Le fou dévoile la vérité élémentaire de 'homme : elle le réduit à ses désirs primitifs, à ses mécanismes simples, aux déterminations les plus pressantes de son corps. (...) Mais le fou dévoile la fin terminale de l'homme : il montre jusqu'où ont pu le pousser les passions, la vie de société, tout ce qui l'écarte d'une nature primitive qui ne connaît pas la folie. Celle-ci est toujours liée à une civilisation et à son malaise ${ }^{17}$. „ De ce point de vue, on peut dire que les conduites à risque, en tant que formes de folies sociétales caractéristiques des sociétés postmodernes constituent une négation radicale des idéaux de la modernité et correspondent à une redécouverte des passions, comme véritable soubassement des comportements humains. Elles représentent une réfutation catégorique des conceptions linéaires des philosophies de l'histoire pour lesquelles « l'instinct de la raison trouve aussi dans sa recherche seulement la raison elle-même » 18 .

De même, les conduites à risque opposent une résistance à l'idéologie bourgeoise fondée sur le substantialisme de la conscience. Celui-ci implique la domination du monde et de soi, la suprématie du libre-arbitre ou de ce que Hans Jonas appelle le principe de responsabilité. Les conduites à risque nous permettent de redécouvrir l'animal qui veille en nous. Rappelons, dans ce contexte, ce que nous enseigne Hermann Hesse : «On ne peut vivre intensément qu'aux dépens du moi. Le bourgeois, précisément, n'apprécie rien autant que le moi (un moi qui n'existe, il est vrai, qu'à l'état rudimentaire). Ainsi, au détriment de l'intensité, il obtient la conservation et la sécurité ; au lieu de la folie en Dieu, il récolte la tranquillité de la conscience ; au lieu de la volupté, le confort ; au lieu de la liberté, l'aisance ; au lieu de l'ardeur mortelle, une température agréable. Le bourgeois, de par sa nature, est un être doué d'une faible vitalité, craintif, effrayé de tout abandon, facile à gouverner. C'est pourquoi, à la place de la puissance, il a mis la majorité ; à la place de la force, la loi ; à la place de la responsabilité, le droit de vote 19. "

L'exemple des conduites à risque est paradigmatique dans la mesure où il instruit une dialectique entre l'individuel et le collectif. Les conduites dangereuses, tant pour la vie d'autrui que pour soi-même, semblent porter la marque de l'indi-

17. M. Foucault, Histoire de la folie à l'âge classique, Paris, Gallimard, 1976, p. 538. 18. G. W. F. Hegel, La phénoménologie de l'esprit, tome I, Paris, Aubier, 1987, p. 219. 19. H. Hesse, "Traité du loup des steppes. Seulement pour les fous ", in Le loup des steppes, Paris, Le Livre de Poche, 1976, pp. I-XXXI, p. XV pour la citation. vidualisme moderne. Cependant, elles sont à comprendre sous le prisme du collectif. Durkheim nous avait incités à appréhender le suicide, qui émane d'une volonté et d'une décision individuelles, comme une manifestation du collectif. Les conduites à risque se propagent dans la société sous la forme de la contagion, dans un mouvement d'amplification issu d'une manifestation collective, qui peut être l'ambiance d'une brasserie ou d'une cave à vin, ou encore le partage d'émotions communes dans une situation de danger, subie ou délibérément provoquée. On peut s'en remettre aux propos de Max Scheler : « Dans toutes les excitations collectives, et même lors de la formation de ce qu'on appelle l'"opinion publique", c'est surtout la réciprocité de cette contagion cumulative qui provoque le mouvement émotionnel collectif et produit cette situation singulière dans laquelle la "masse" agit sans tenir compte des intentions des individus qui la composent et accomplit des choses dont aucun de ces individus ne veut se reconnaître "responsable" parce qu'il ne les a pas "voulues". C'est, en fait, le processus de la contagion lui-même qui produit des fins et des buts situés au-delà des intentions de chacun des individus qui composent la masse 20 ."

On peut dire que les conduites à risque comme toute forme de manifestation ritualisée de la furie sont socialement utiles. La violence dans les stades de football, par exemple, sert d'exutoire à l'irrépressible agressivité qui est en nous. Elle joue le même rôle que la soupape de sécurité d'une cocotte-minute, permettant à la vapeur de s'échapper à petites doses afin qu'elle n'explose pas. C'est en ce sens précis que Durkheim affirmait que la violence qui s'est historiquement exprimée dans les croisades ou les révolutions ne devait pas être jugée du point de vue moral, mais du point de vue de ses effets sur la morale. En effet, la transgression des interdits sert à renforcer les sentiments communs qui donnent vie aux différents groupes sociaux et garantissent leur cohésion.

Même si la société industrielle avancée est centrée sur le principe de la "répartition des risques" 21 , à savoir sur leur potentialisation par le biais de la modernisation, au point que ces risques deviennent un des éléments moteurs constitutifs de nos sociétés, l'homme continuera à promouvoir des attitudes se rapportant à l'ordalie. Cela veut dire que la totalité du groupe ou une partie de ses membres confieront leur vie, et même leur survie au hasard, à la fortune, au destin, au jugement divin. Même si les conduites à risque sont de nature essentiellement individualiste et constituent "un acte solitaire et imprévisible dans son jaillissement " 22 , dépouillé du ritualisme de la communauté, elles contribuent, cependant, comme cela se produit dans les sociétés primitives, à restaurer "une relation au monde plus propice » 23 . La dialectique entre l'ordre et le désordre, entre la perte de soi et

20. M. Scheler, Nature et formes de la sympathie, Paris, Payot \& Rivages, 2003, p. 66. 21. U. Beck, La société du risque. Sur la voie d'une autre modernité, Paris, Flammarion, 2001, p. 35.

22. D. Le Breton, Conduites à risque. Des jeux de mort au jeu de viure, Paris, PUF, 2004, p. 112 .

23. Ibid., p. 110

Sociétés $n^{\circ} 114-2011 / 4$

Sociétés $n^{\circ} 114-2011 / 4$ 
la rencontre avec les autres constitue précisément la condition de la survie individuelle et collective. Les conduites à risque sont autant de formes de ritualisation travers lesquelles l'individu cherche à se socialiser, à former une relation sociale, à entrer en communauté, à faire corps avec les membres de sa tribu. Cette socialisation peut prendre la voie de la déviance ou de la perversion. En tout cas, elle est le prétexte d'une participation à des rituels qui permettent aux jeunes la "mise en scène sociale de leur personnalité » 24

Les conduites à risque endossent les formes les plus diverses : le refus délibéré de préservatifs lors des rapports sexuels, l'usage de drogues, les beuveries (qui son l'objet de compétitions organisées), le saut à l'élastique, le base-jump, qui consiste à se lancer du haut d'un immeuble avec un parachute, le canyoning, qui consiste à descendre des rivières serpentant au milieu de falaises abruptes, avec son lot de cascades et d'obstacles, la plongée en apnée, la pratique compulsive des sports radicaux (de la pratique de la break dance à l'escalade dans des conditions extrêmes), la consommation de substances neurotoxiques aux effets le plus souvent méconnus et incontrôlés. Ces conduites constituent autant de formes de résistance au délire de l'impérialisme de la morale, qui commence précisément, comme l'a remarquablement indiqué Ruwen Ogien, quand celle-ci s'occupe des dommages qu'on se cause à soi-même au lieu de traiter des préjudices causés à autrui. L'auteur s'en explique on ne peut plus clairement : "Imaginez un monde dans lequel on pourrait vous juger "immoral", non seulement pour vos actions, mais aussi pour vos pensées, vos désirs, vos fantasmes ou vos traits de "caractère". Non seulement pour ce que vous faites aux autres, mais aussi pour ce que vous vous faites à vous-même. Non seulement pour ce que vous faites délibérément, en toute connaissance de cause, mais aussi pour ce qui vous arrive un peu par hasard. (...) Qui aimerait viure dans un tel monde, où rien de ce qu'on est, pense ou ressent, aucune de nos activités, fût-elle la plus solitaire, n'échappe au jugement moral ? 25.

Les jeux qui sont pratiqués dans les écoles, c'est-à-dire au sein même d'institutions détentrices et dispensatrices du moralisme le plus affuté, et qui permettent aux enfants de compenser l'imposition d'un monde qui leur échappe complètement par la sensation de disposer librement de leur corps, de jouer avec ses mouvements et, par conséquent, avec leur propre vie, sont tout à fait symptomatiques de l'immoralisme éthique et esthétique postmoderne. Il semblerait que le corps en mouvement condense en lui les nouvelles formes d'utopies, indices d'une socialité intensément vécue au quotidien.

Une petite incursion dans la Toile nous a permis de recenser quelques-uns de ces jeux qui brutalisent et transfigurent les corps. En premier lieu, les jeux de désoxygénation. C'est le cas du " jeu du foulard ", dont le but réside dans la recherche de sensations euphoriques et dont le principe est de provoquer un évanouissement par strangulation, en raison du manque d'imigation en oxygène dans le

24. D. Jeffrey, Éloge des rituels, Sainte-Foy, Les Presses de l'Université Laval, 2005, p. 96. 25. R. Ogien, L'éthique aujourd'hui. Maximalistes et minimalistes, Paris, Folio, 2007, p. 11.

Sociétés $n^{\circ} 114-2011 / 4$

cerveau. Ce jeu comporte de nombreuses dénominations, qui varient selon les lieux cosmos, été indien, rêve bleu. Comme variante à ce jeu, on a le jeu de la tomate, où l'on bouche le nez jusqu'à ce que l'on rougisse, le jeu du sternum (ou de la grenouille) qui consiste à bloquer la respiration en comprimant fortement la poitrine. C'est encore le cas du " jeu du pulvérisateur ", dont le but est de déformer la voix en inhalant le produit d'un aérosol quelconque, ce qui peut créer un œedème pulmonaire.

En second lieu, le recensement de ces artifices rend compte des jeux d'attaque. C'est le cas du « jeu de la canette » qui a pour but de prouver sa force. Dans ce jeu, les enfants forment un cercle et une canette est jetée dans la direction de l'un des participants. Si l'enfant n'est pas capable de l'attraper, il est battu. Il est entendu que la victime d'aujourd'hui pourra devenir le bourreau du lendemain. Ce jeu, à l'instar des autres, comporte différents noms : le bouc émissaire, le jeu du jugement, le jeu des chaussures. Le " jeu de la boule ", qui consiste à battre un camarade de classe, à titre gratuit, et ainsi à tester les limites de son endurance est du même type. Cela est également vrai pour le " jeu du taureau ", dans lequel une bande de jeunes fonce têtes baissées vers un enfant esseulé. C'est encore le cas pour le « jeu de Beyrouth»: un enfant demande à un autre quelle est la capitale du Liban. S'il est incapable de répondre, on lui fustigera les parties génitales.

En troisième lieu, on peut dénombrer des jeux de pure violence sur soi-même, comme le jackass, à l'instar de ce jeune Américain qui a bouché son anus avec une colle extra-forte.

Même lorsque les dangers sont minimisés, condamnés à la banalisation et à la programmation, comme dans le cas des marathons dans le désert du Sahara, des retraites en Amazonie, du trekking dans l'Himalaya, il s'agit pour les individus de vivre un événement d'exception qui, lorsqu'il est partagé avec d'autres, conduit à « la formation d'une communitas, dont les actions réciproques sont bâties sur un risque initiatique 226

En tout cas, nous ne pouvons pas circonscrire l'explication des conduites à risque au malaise individuel, comme conséquence d'une absence de valeurs susceptibles d'agréger les individus dans des sociétés hautement individualisées. Ni même recourir à la thèse d'une désagrégation de la structure familiale, d'une toujours plus grande irresponsabilité des familles en matière de transmission des valeurs spirituelles, morales ou civiques. Pour comprendre les risques encourus de façon volontaire par les jeunes, nous devons avant tout tenir compte du mimétisme inhérent à ces comportements. Le phénomène de l'imitation a trait au suicide, à la consommation de drogues, aux jeux dangereux. Le groupe est à la fois une source d'émulation pour les conduites à risque et un élément de protection pour les individus. Dans le groupe, l'individu se sent stimulé et comme invincible. Ainsi, les sensations d'autoréalisation sont démultipliées au contact des autres et finissent par fusionner au sein

6. Marthélemy, "L'engouement pour les raids-aventure ou la société du risque transfigurée par le destin ", Sociétés, 2002, 77 (3), p. 91.

Sociétés $n^{\circ} 114-2011 / 4$ 
de relations sociales caractérisées par l'intersubjectivité et l'intercorporalité. En d'autres termes, l'expérience vécue du risque renforce la valeur sociale des individus et favorise leur intégration dans le groupe.

D'un point de vue sociologique, il serait tout à fait judicieux de concevoir les maux sociaux et moraux de la même manière que nous concevons les maux naturels, c'est-à-dire comme une expression du hasard ou de la contingence plutôt que de l'intentionnalité et faire ainsi la part belle à cette « ruse métaphysique qui consiste à se débarrasser d'une partie de la responsabilité du mal en en faisant un destin $" 27$. Nous savons que les individus extériorisent leur besoin de vivre dans l'excès en adoptant des conduites routières dangereuses, à l'exemple du « chicken game ", où deux voitures foncent vers le précipice dans le but de révéler le pilote le plus courageux, celui qui s'extrait en dernier de la voiture juste avant la chute finale, dans la scène du film La fureur de viure, immortalisée par James Dean. Là encore, on pourrait adopter le point de vue de la « dromoscopie - ce phénomène optique qui inverse le sens des abords de la route, avec ses arbres qui ont l'air de se précipiter sur le pare-brise avant de disparaître dans la lunette arrière alors qu'en réalité c'est l'inverse qui se produit » 28

On retrouve le même ordre d'idées chez Olivier Sirost, dans son analyse des peurs et des risques, en particulier quand il montre que la sublimation de la peur constitue un "point d'inflexion du sentiment de "risque" „29. C'est de ce point de vue qu'il analyse le glissement, au XVIIle siècle, des sentiments à l'égard des éléments naturels, comme la mer et la montagne, perçus à la fois comme sources de dangers et de peurs, mais aussi comme vecteur d'attraction, dont le romantisme naissant s'est fait l'écho. À la suite des études d'Alain Corbin sur les rapports entre le rivage et la mer, Sirost souligne un changement de paradigme dans la perception des dangers liés aux aventures nautiques : " à l'égard de la mer, le rivage est un territoire qui se constitue au cours des XVIIIle et XIXe siècles en passant du statut d'abysse des peurs à celui de grève des plaisirs. Le changement s'opère par une réorientation esthétique des sentiments où la peur et le frisson s'intègrent au sublime. La scène maritime qui avive ces émotions change son cadre perceptif. Désormais la mer s'appréhende à partir du point fixe et sécurisant qu'est le rivage, et non plus en tant que "mobilis in mobile" 30 . "On retrouve bien cette conjugaison d'effroi et d'agrément, de répulsion et d'attraction dans les conduites à risque actuelles.

Amadouer les nombreux dangers induits par l'indomptable "vouloir-vivre" (Maffesoli) - dont témoignent des expressions telles que toucher le fond, se frotter au danger, vivre dans l'excès, s'éclater, s'exploser, se défoncer, courtiser la mort en acceptant le destin, voilà le secret du bon usage des risques. Du bon usage aussi

27. J.-P. Dupuy, Petite métaphysique des tsunamis, Paris, Seuil, 2005, p. 27.

28. P. Virilio, L'accident originel, Paris, Galilée, 2005, p. 141.

29. O. Sirost, "Se mettre à l'abri ou jouer sa vie? Éléments d'une culture sociale du

risque ", Sociétés, 2002, 77 (3), p. 6.

30. Ibid. des catastrophes, dont le médiologue Régis Debay s'est fait le porte-parole. C'est ainsi qu'il nous enseigne l'art " d'éviter les envolées en apprenant comment ne pas devenir prophète (...) Avec lui [le médiologue], on ne va jamais plus loin et toujours plus bas. À même le soubassement, le vulgaire et l'infime. Pas de voie royale, nulle conception générale de l'univers. Pas un traître mot avec majuscule, rien sur Amour, Justice et Bonheur ${ }^{31}$. "

Les conduites à risque nous renvoient pour ainsi dire à la concrétude du quotidien. En plus de représenter l'une des rares sources de liberté dans une société qui tend invariablement à l'uniformité, elles ont l'incomparable mérite de poser la question de l'altérité, une altérité qui est en nous et autour de nous. La question qui se pose alors au sociologue est de se montrer à la hauteur du quotidien et d'intégrer à sa réflexion cette réalité indélébile de la vie qu'est l'altérité, cette part maudite qui tour à tour s'incarne dans les figures du mal, de la violence, de la cruauté, de la furie, de la folie: "Dialogique de la pars destruens et la pars construens. Destructions et constructions vont de pair. Et l'art du savoir est bien de s'ajuster à l'art de vivre reposant sur une telle dialogie ${ }^{32}$. "

\section{Bibliographie}

Barthélemy M., « L'engouement pour les raids-aventure ou la société du risque transfigurée par le destin ", in Sociétés, 2002, 77 (3), pp. 83-93.

Bataille G., L'érotisme, Paris, Les Éditions de Minuit, 1985.

Beck U., La société du risque. Sur la voie d'une autre modernité, Paris, Flammarion, 2001.

Debray R., Du bon usage des catastrophes, Paris, Gallimard, 2011.

Delumeau J., La peur en Occident (XIVe-XVIII siècles), Paris, Hachette/Pluriel, 1985.

Dupuy J.-P., Petite métaphysique des tsunamis, Paris, Seuil, 2005.

Empoli G. da, La peste et l'orgie, Paris, Grasset, 2006.

Ewald F., L'État providence, Paris, Grasset, 1994.

Foucault M., Histoire de la folie à l'âge classique, Paris, Gallimard, 1976.

Gadamer H.-G. Philosophie de la santé, Paris, Grasset \& Fasquelle et Mollat, 1998.

Hegel G. W. F., La phénoménologie de l'esprit, 2 tomes, Paris, Aubier, 1987.

Hesse H., Le loup des steppes, Paris, Le Livre de Poche, 1976.

Horkheimer M., Éclipse de la raison, suivi de Raison et conservation de soi, Paris, Payot, 1974.

Jeffrey D., Éloge des rituels, Sainte-Foy, Les Presses de l'Université Laval, 2005.

Le Breton D. La peau et la trace. Sur les blessures de soi, Paris, Métailié, 2003.

Le Breton D., Conduites à risque. Des jeux de mort au jeu de viure, Paris, PUF, 2004

Maffesoli M., Logique de la domination, Paris, PUF, 1976.

Maffesoli M., Le réenchantement du monde. Une éthique pour notre temps, Paris, La Table Ronde, 2007.

31. R. Debray, Du bon usage des catastrophes, Paris, Gallimard, 2011, p. 107

31. R. Debray, Du bon usage des catastrophes, Paris, Gallimard, $2011, \mathrm{p}$. Table Ronde, 2007, p. 30. 
Ogien R., L'éthique aujourd'hui. Maximalistes et minimalistes, Paris, Folio, 2007. Ortega y Gasset J., La révolte des masses, Paris, Gallimard, 1967.

Peretti-Watel P., La société du risque, Paris, La Découverte \& Syros, 2001

Scheler M., Nature et formes de la sympathie, Paris, Payot \& Rivages, 2003.

Sirost $\mathrm{O}$., "Se mettre à l'abri ou jouer sa vie ? Éléments d'une culture sociale du risque », Sociétés, 2002, 77 (3), pp. 5-15.

Taguieff P.-A., Du progrès. Biographie d'une utopie moderne, Paris, E.J.L, 2001

Virilio P., L'accident originel, Paris, Galilée, 2005.

Weber M., Le savant et le politique, Paris, Union Générale d’Éditions, 1974 